\title{
COFINAL FAMILIES OF COMPACT SUBSETS OF AN ANALYTIC SET
}

\author{
HOWARD BECKER
}

(Communicated by Dennis K. Burke)

\begin{abstract}
We consider a question of van Douwen regarding the minimum cardinality of cofinal families of compact sets in certain topological spaces. We show that the question cannot be answered in ZFC.
\end{abstract}

The cardinal $\mathfrak{d}$ is defined as follows.

$$
\begin{aligned}
\mathfrak{d}=\min \left\{|\mathscr{D}|: \mathscr{D} \subset \omega^{\omega} \text { and for all } f \in \omega^{\omega}, \text { there is a } g \in \mathscr{D}\right. \\
\text { such that for all but finitely many } n, g(n)>f(n)\} .
\end{aligned}
$$

Clearly $\aleph_{1} \leq \mathfrak{d} \leq \mathfrak{c}$, and $\operatorname{cof}(\mathfrak{d})>\omega$. Hechler [5] showed that nothing else about the size of $\mathfrak{d}$ can be proved in ZFC.

For any topological space $X$, let $\mathscr{K}(X)$ be the space of all non-empty compact subsets of $X$, with the Vietoris topology. Define the cardinal $\operatorname{cof}(\mathscr{K}(X))$ as follows.

$$
\begin{aligned}
\operatorname{cof}(\mathscr{K}(X))=\min \{|\mathscr{L}|: \mathscr{L} \subset \mathscr{K}(X) \text { and for all compact } K \subset X, \\
\text { there is an } L \in \mathscr{L} \text { such that } K \subset L\} .
\end{aligned}
$$

Note that $\mathfrak{d}=\operatorname{cof}\left(\mathscr{K}\left(\omega^{\omega}\right)\right)$, where $\omega^{\omega}$ has the usual topology.

Van Douwen [2] considered the problem of calculating $\operatorname{cof}(\mathscr{K}(X))$ for various separable metrizable spaces $X$. He showed that for any such $X$, if $X$ is analytic $\left(\Sigma_{1}^{1}\right)$ but not $\sigma$-compact, then $\operatorname{cof}(\mathscr{K}(X)) \geq \mathfrak{d}$. He asked ([1], [2, 8.11]) two questions: If $X$ is separable and metrizable and analytic (or at least absolutely Borel), is $\operatorname{cof}(\mathscr{K}(X)) \leq \mathfrak{d}$ ? I thank Peter Nyikos for bringing these questions to my attention.

Van Engelen [3] gave a positive answer to the Borel question. In fact, he proved a stronger statement: If $X$ is any coanalytic $\left(\Pi_{1}^{1}\right)$ set in a Polish space, then $\operatorname{cof}(\mathscr{K}(X)) \leq \mathfrak{d}$.

Clearly it is consistent with ZFC that van Douwen's analytic question also has a positive answer-for example, if the continuum hypothesis holds. The purpose of this paper is to show that a negative answer is also consistent, hence the question cannot be decided in ZFC.

Received by the editors August 17, 1988.

1980 Mathematics Subject Classification (1985 Revision). Primary 54A35.

The author's research was partially supported by NSF Grant DMS-8601731. 
Theorem. $\operatorname{Con}(Z F C)$ implies $\operatorname{Con}\left(Z F C+\mathfrak{d}=\aleph_{1}+\right.$ There is a $\Sigma_{1}^{1}$ set $X \subset 2^{\omega}$ such that $\left.\operatorname{cof}(\mathscr{K}(X))=\aleph_{2}=\mathfrak{c}\right)$.

For more information on the topological matters, see van Douwen [2]; on $\Sigma_{n}^{1}$ and $\Pi_{n}^{1}$ sets, see Moschovakis [9]; on consistency proofs, see Jech [6].

Lemma 1 (Martin-Solovay [8]; see [4, 23J and 23N(g)]). Con $(Z F C)$ implies $\operatorname{Con}\left(Z F C+\mathfrak{c}=\aleph_{2}+\right.$ Every set of cardinality $\aleph_{1}$ in every Polish space is $\left.\mathbf{\Pi}_{1}^{1}\right)$.

Lemma 2 (Hechler [5]; see [6, p. 260]). There exists a notion of forcing which preserves cardinals, preserves the value of $\mathfrak{c}$, and makes $\mathfrak{d}=\aleph_{1}$.

Let $\boldsymbol{\pi}_{1}^{1}=\sup \left\{\alpha \in\right.$ Ord: $\alpha$ is the rank of a $\Pi_{1}^{1}$

well-founded relation on $2^{\omega}$ \}.

By the Kunen-Martin Theorem (see [9, 2G.4]), $\boldsymbol{\pi}_{1}^{1} \leq \aleph_{2}$.

Lemma 3. $\operatorname{Con}(Z F C)$ implies $\operatorname{Con}\left(Z F C+\mathfrak{d}=\aleph_{1}+\pi_{1}^{1}=\aleph_{2}=\mathfrak{c}\right)$.

Proof. If every set of cardinality $\aleph_{1}$ is $\Pi_{1}^{1}$, then there are $\Pi_{1}^{1}$ well-orderings of every order-type less than $\omega_{2}$, so clearly $\boldsymbol{\pi}_{1}^{1}=\aleph_{2}$. Therefore, by Lemma 1 , $\operatorname{Con}(\mathrm{ZFC})$ implies $\operatorname{Con}\left(\mathrm{ZFC}+\boldsymbol{\pi}_{1}^{1}=\aleph_{2}=\mathfrak{c}\right)$. We next show: $\operatorname{Con}\left(\mathrm{ZFC}+\boldsymbol{\pi}_{1}^{1}=\right.$ $\left.\aleph_{2}=\mathfrak{c}\right)$ implies Con $\left(Z F C+\mathfrak{d}=\aleph_{1}+\pi_{1}^{1}=\aleph_{2}=\mathfrak{c}\right)$. Let $M \models\left(Z F C+\pi_{1}^{1}=\aleph_{2}=\right.$ c), let $P$ be a notion of forcing satisfying Lemma 2 , and let $N$ be $P$-generic over $M$. Thus $N \models\left(Z F C+\mathfrak{d}=\aleph_{1}+c=\aleph_{2}\right)$. Let $\lambda<\aleph_{2}^{M}=\aleph_{2}^{N}$; to complete the proof it will suffice to show that $\lambda<\left(\boldsymbol{\pi}_{1}^{1}\right)^{N}$. Since $M \models\left(\boldsymbol{\pi}_{1}^{1}=\aleph_{2}\right)$, there is, in $M$, a wellfounded $\Pi_{1}^{1}$ binary relation $R$ on $2^{\omega}$ of rank at least $\lambda$. Let $\psi$ be a $\Pi_{1}^{1}$-formula with two free variables (and a real parameter from $M$ ) which defines $R$. The statement "The relation $\psi$ is wellfounded" is $\Pi_{2}^{1}$, so by the Shoenfield Absoluteness Theorem, it is true in $N . \psi$ itself is, of course, also absolute, so $R \subset R^{\prime}$, where

$$
R^{\prime}=\{(x, y) \in N \times N: N \models \psi(x, y)\} .
$$

So $\operatorname{rank}\left(R^{\prime}\right) \geq \operatorname{rank}(R)$. Hence, in $N$, there is a $\Pi_{1}^{1}$ wellfounded relation of rank at least $\lambda$. That is, $\lambda<\left(\boldsymbol{\pi}_{1}^{1}\right)^{N}$.

For any topological space $Y$, let $\mathrm{kc}(Y)$ denote the compact covering number of $Y$, that is,

$$
\min \{|\mathscr{L}|: \mathscr{L} \subset \mathscr{K}(Y) \text { and } \bigcup \mathscr{L}=Y\} .
$$

Lemma 4. If $\boldsymbol{\pi}_{1}^{1}=\aleph_{2}$, then there is a $\Pi_{2}^{1}$ set $Y \subset 2^{\omega}$ such that $\operatorname{kc}(Y) \geq \aleph_{2}$. Proof. Let $U \subset 2^{\omega} \times\left(2^{\omega} \times 2^{\omega}\right)$ be universal for $\Pi_{1}^{1}$ subsets of $2^{\omega} \times 2^{\omega}$, i.e., $U$ is $\Pi_{1}^{1}$ and every $\Pi_{1}^{1}$ set in $2^{\omega} \times 2^{\omega}$ is equal to $U_{x}=\{(y, z):(x, y, z) \in U\}$ for some $x$. Let

$$
Y=\left\{x \in 2^{\omega}: \text { The binary relation } U_{x} \text { is wellfounded }\right\} .
$$


$Y$ is $\Pi_{2}^{1}$. Clearly $\sup \left\{\operatorname{rank}\left(U_{x}\right): x \in Y\right\}=\pi_{1}^{1}=\aleph_{2}$. We claim that for any $\Pi_{1}^{1}$ set $Z \subset Y, \sup \left\{\operatorname{rank}\left(U_{x}\right): x \in Z\right\}<\pi_{1}^{1}$. Assuming this claim, for any collection $\mathscr{L}$ of $\aleph_{1}$ compact (or even $\Pi_{1}^{1}$ ) subsets of $Y, \sup \left\{\operatorname{rank}\left(U_{x}\right): x \in\right.$ $\cup \mathscr{L}\}<\aleph_{2}$ hence $\mathscr{L}$ cannot cover $Y$. To prove the claim, let $Z \subset Y$ be $\Pi_{1}^{1}$. Define $R \subset\left(2^{\omega} \times 2^{\omega}\right) \times\left(2^{\omega} \times 2^{\omega}\right)$ as follows:

$$
\left((x, y),\left(x^{\prime}, y^{\prime}\right)\right) \in R \text { iff }\left[x=x^{\prime} \text { and } x \in Z \text { and }\left(x, y, y^{\prime}\right) \in U\right] .
$$

$R$ is a $\Pi_{1}^{1}$ binary relation on $2^{\omega} \times 2^{\omega}$, and it is wellfounded. It is easy to see that the rank of $R$ is $\sup \left\{\operatorname{rank}\left(U_{x}\right): x \in Z\right\}$. Since $R$ is $\Pi_{1}^{1}$, this rank must be less than $\pi_{1}^{1}$.

Lemma 5 (van Engelen [3]). For any $X \subset 2^{\omega}, \operatorname{cof}(\mathscr{K}(X))=\mathrm{kc}(\mathscr{K}(X))$.

Let $\Gamma$ be either $\Sigma_{1}^{1}$ or $\Pi_{2}^{1}$, and let $E$ be a 0 -dimensional compact metrizable space. A pointset $Y \subset E$ is called $\Gamma$-hard if for every 0-dimensional compact metrizable space $E^{\prime}$, and every $\Gamma$ set $Z \subset E^{\prime}$, there is a continuous function $f: E^{\prime} \rightarrow E$ with $Z=f^{-1}[Y]$. A set is $\Gamma$-complete if it is a $\Gamma$ set which is $\Gamma$-hard. Note that $\Gamma$-complete sets exist; for example, any universal set is complete.

Lemma 6 (Kechris-Louveau-Woodin [7, p. 266]). For any $X \subset 2^{\omega}$, if $X$ is $\Sigma_{1}^{1}$-hard, then $\mathscr{K}(X)$, considered as a pointset in the space $\mathscr{K}\left(2^{\omega}\right)$, is $\Pi_{2}^{1}$-hard.

Lemma 7. For any cardinal $\kappa$, if there exists a $\Pi_{2}^{1}$ set $Y \subset 2^{\omega}$ with $\mathrm{kc}(Y) \geq \kappa$, then there exists a $\Sigma_{1}^{1}$ set $X \subset 2^{\omega}$ with $\operatorname{cof}(\mathscr{K}(X)) \geq \kappa$.

Proof. Let $Y \subset 2^{\omega}$ be $\Pi_{2}^{1}$ with $\operatorname{kc}(Y) \geq \kappa$. Let $X \subset 2^{\omega}$ be any $\Sigma_{1}^{1}$ complete set. By Lemma $6, \mathscr{K}(X)$ is $\Pi_{2}^{1}$-hard, so there exists a continuous $f: 2^{\omega} \rightarrow \mathscr{K}\left(2^{\omega}\right)$ such that $f^{-1}[\mathscr{K}(X)]=Y$. If $\mathscr{L} \subset \mathscr{K}(\mathscr{K}(X))$ covers $\mathscr{K}(X)$, then $\left\{f^{-1}[K]: K \in \mathscr{L}\right\}$ covers $Y$, so $\mathrm{kc}(\mathscr{K}(X)) \geq \mathrm{kc}(Y)$. By Lemma $5, \operatorname{cof}(\mathscr{K}(X))=\mathrm{kc}(\mathscr{K}(X)) ;$ hence $\operatorname{cof}(\mathscr{K}(X)) \geq \kappa$.

Lemma 8. If $\pi_{1}^{1}=\aleph_{2}$, then there is a $\Sigma_{1}^{1}$ set $X \subset 2^{\omega}$ such that $\operatorname{cof}(\mathscr{K}(X)) \geq$ $\aleph_{2}$.

Proof. Lemmas 4 and 7.

The theorem follows from Lemmas 3 and 8 .

\section{REFERENCES}

1. E. K. van Douwen, Problem 11, Topology Proc. 6 (1981), 443.

2. The integers and topology, in Handbook of Set Theoretic Topology, (K. Kunen and J.

E. Vaughan, eds.) North-Holland, Amsterdam, 1984, 111-167.

3. F. van Engelen, Cofinal families of compacta in separable metric spaces, Proc. Amer. Math. Soc. 104 (1988), 1271-1273.

4. D. H. Fremlin, Consequences of Martin's axiom, Cambridge Univ. Press, Cambridge, 1984. 
5. S. H. Hechler, On the existence of certain cofinal subsets of $\omega^{\omega}$, in Axiomatic Set Theory, (T. J. Jech, ed.), Proc. Symp. Pure Math., vol. 13, Part 2, Amer. Math. Soc., Providence, R.I., 1974, pp. 155-173.

6. T. J. Jech, Set theory, Academic Press, 1978.

7. A. S. Kechris, A. Louveau, and W. H. Woodin, The structure of $\sigma$-ideals of compact sets, Trans. Amer. Math. Soc. 301 (1987), 263-288.

8. D. A. Martin and R. M. Solovay, Internal Cohen extensions, Annals Math. Logic 2 (1970), 143-178.

9. Y. N. Moschovakis, Descriptive set theory, North-Holland, Amsterdam, 1980.

Department of Mathematics, University of South Carolina, Columbia, South CAROLINA 29208 